\title{
Three novel mutations of the PAX6 gene in Japanese aniridia patients
}

\author{
Toshio Kawano Chunxia Wang Y Yoshihiro Hotta $\cdot$ Miho Sato $\cdot$ Emi Iwata-Amano • \\ Akiko Hikoya - Naoya Fujita $\cdot$ Norihisa Koyama Shoichiro Shirai $\cdot$ Noriyuki Azuma \\ Masafumi Ohtsubo $\cdot$ Nobuyoshi Shimizu $\cdot$ Shinsei Minoshima
}

Received: 18 February 2007/Accepted: 17 April 2007/Published online: 14 June 2007

(C) The Japan Society of Human Genetics and Springer 2007

\begin{abstract}
Mutations in the PAX6 gene of Japanese aniridia patients were analyzed. Four types of mutations including one known (474delC) and three novel (786_787ins10,
\end{abstract}

Electronic supplementary material The online version of this article (doi:10.1007/s10038-007-0153-2) contains supplementary material, which is available to authorized users.

T. Kawano - C. Wang · Y. Hotta · M. Sato · A. Hikoya

Department of Ophthalmology,

Hamamatsu University School of Medicine,

Hamamatsu, Japan

C. Wang $\cdot$ M. Ohtsubo $\cdot$ S. Minoshima $(\bowtie)$

Medical Photobiology Department, Photon Medical Research

Center, Hamamatsu University School of Medicine,

1-20-1 Handayama, Hamamatsu 431-3192, Japan

e-mail: mino@hama-med.ac.jp

E. Iwata-Amano

Department of Ophthalmology,

Nagoya University Graduate School of Medicine,

Nagoya, Japan

N. Fujita $\cdot$ N. Koyama

Department of Pediatrics, Toyohashi Municipal Hospital,

Toyohashi, Japan

S. Shirai

Department of Ophthalmology, Toyohashi Municipal Hospital,

Toyohashi, Japan

N. Azuma

Department of Ophthalmology,

National Center for Child Health and Development,

Tokyo, Japan

N. Shimizu

Department of Molecular Biology,

Keio University School of Medicine,

Tokyo, Japan 678_688del11 and 572_575delAATCins14) were found in six patients from four families. A patient with the mutation 572_575delAATCins14 also manifested VATER association. This is the first case of aniridia accompanied by VATER association. All of mutations found in this study are frameshift type, resulting in premature termination of translation. The database for PAX6 gene mutation has been made using a graphical data display system MutationView (http://mutview.dmb.med.keio.ac.jp/).

Keywords Aniridia P PAX6 - Mutation - Database . VATER association

\section{Introduction}

Aniridia is a panocular autosomal dominant disorder in which the development of the iris, cornea, lens, angle, and retina is disturbed. In the general population, aniridia occurs at a frequency of 1 in 50,000-100,000 (Elsas et al. 1997). Aniridia is often caused by mutations of the PAX6 gene, located on the band p13 of human chromosome 11 (Ton et al. 1991). The PAX6 gene encodes a transcription regulator that recognizes target genes through the paired domain and is involved in ocular morphogenesis. Numerous mutations have been detected in patients with aniridia (Prosser et al. 1998) and the Human PAX6 Mutation Database (http://www.pax6.hgu.mrc.ac.uk/) collected approximately 300 cases with 200 different mutations. Of these, 26 cases with 19 different types of mutations are from Japanese patients (Azuma et al. 1996, 1999, 2003; Sonoda et al. 2000; Yasuda et al. 2002). Here, we report additional mutations of the PAX6 gene in Japanese aniridia patients, including three novel types of mutations. 


\section{Materials and methods}

Patients

Six patients analyzed in this study were members of four unrelated families (Table 1; Supplemental Fig. 1). Patient AN011 and his mother AN012 are from family 1, in which no other patients were found with aniridia or related disorders including generation I. It is notable that their aniridia phenotype was mild. Patient AN021 is from family 2, which includes ten patients for four generations. Patient AN031 is from family 3, and is apparently a sporadic case of aniridia. Two members of generation I and III had color blindness. Patients AN041 and AN042 are from family 4. AN041 developed anal atresia, hydronephrosis, right radial dysplasia and loss of the right thumb. Consequently, AN041 was diagnosed as VATER association (Quan and Smith 1972) with aniridia. However, patient AN042, a younger brother of AN041, showed only aniridia. Three members of generation I and II in family 4 showed strabismus, whereas the mother of AN041 and AN042 was apparently normal.

\section{DNA samples}

The molecular genetic study was approved by the Institutional Review Board for Human Genetic and Genomic Research of Hamamatsu University School of Medicine, and the procedures conformed to the tenets of the Declaration of Helsinki. High molecular weight genomic DNA was isolated from peripheral blood leukocytes. Informed consent was obtained from the patients and non-patient members of their families.

\section{Direct DNA sequencing}

The genomic regions corresponding to the 13 exons of the PAX6 gene were amplified with PCR using the primers previously described (Glaster et al. 1992). The reaction condition was as follows: initial denaturation at $94^{\circ} \mathrm{C}$ for $10 \mathrm{~min}, 30$ cycles of denaturation at $94^{\circ} \mathrm{C}$ for $30 \mathrm{~s}$, annealing at $60^{\circ} \mathrm{C}$ for $30 \mathrm{~s}$, and extension at $72^{\circ} \mathrm{C}$ for $1 \mathrm{~min}$, with a final extension at $72^{\circ} \mathrm{C}$ for $10 \mathrm{~min}$. The PCR products were separated on $2 \%$ agarose gel electrophoresis and visualized by ethidium bromide staining. Agarose gel blocks containing amplified products were excised and DNAs were purified using QIAquick Gel Extraction Kit (Qiagen). The purified PCR products were sequenced on an Applied Biosystems model 3100 automated sequencer (Perkin-Elmer Applied Biosystems Division, CA) with primers that were used for PCR amplification.

Cloning and sequencing of each allele

In the cases where some sequence abnormality was detected by direct sequencing, two alleles were cloned to examine their sequences separately unless otherwise described. The purified PCR products for direct sequencing were ligated to the 3.9-kb pDrive vector (Qiagen) according to the manufacturer's instructions. The construct was transformed into TOP10 E. coli cells, and plated on $50 \mu \mathrm{g} /$ $\mathrm{ml}$ kanamycin, $80 \mu \mathrm{g} / \mathrm{ml} \mathrm{X-gal} \mathrm{containing} \mathrm{LB} \mathrm{agar} \mathrm{plates.}$ Plasmids were isolated from each colony of the transformed cells by the alkaline lysis method. Sequencing was performed using M13 universal primers.

\section{Results}

Mutation analysis

We carried out a mutation analysis of the $P A X 6$ gene in six patients with aniridia from four families. Four types of mutations including three novel ones were found (Fig. 1; Supplemental Fig. 2). All of these mutations were heterozygous in patients. A summary of the patient diagnoses and mutation types are listed in Table 1.

Patients AN011 (son) and AN012 (mother) had a mutation 474delC in exon 5 (Supplemental Fig. 2A), which results in frameshift and premature termination of translation within the paired domain of PAX6 protein. The mutation was not detected in his unaffected father

Table 1 Mutations in the PAX6 gene of aniridia patients analyzed in this study

\begin{tabular}{llllllll}
\hline Patient ID & Remark & Exon & Domain & Type & Nucleotide change & Consequence & Phenotype \\
\hline AN011 & Son of AN012 & 5 & Paired & Deletion & 474delC & Frameshift & Aniridia \\
AN012 & Mother of AN011 & 5 & Paired & Deletion & 474delC & Frameshift & Aniridia \\
AN021 & & 7 & Link & Insertion & 786_787ins10 & Frameshift & Aniridia \\
AN031 & & 6 & Paired & Deletion & 678_688del11 & Frameshift & Aniridia \\
AN041 & Sister of AN042 & 6 & Paired & Indel & 572_575delAATCins14 & Frameshift & Aniridia, VATER asoociation \\
AN042 & Younger brother of AN041 & 6 & Paired & Indel & 572_575delAATCins14 & Frameshift & Aniridia \\
\hline
\end{tabular}

These mutations are being submitted to The Human PAX6 Mutation Database of MRC, UK 


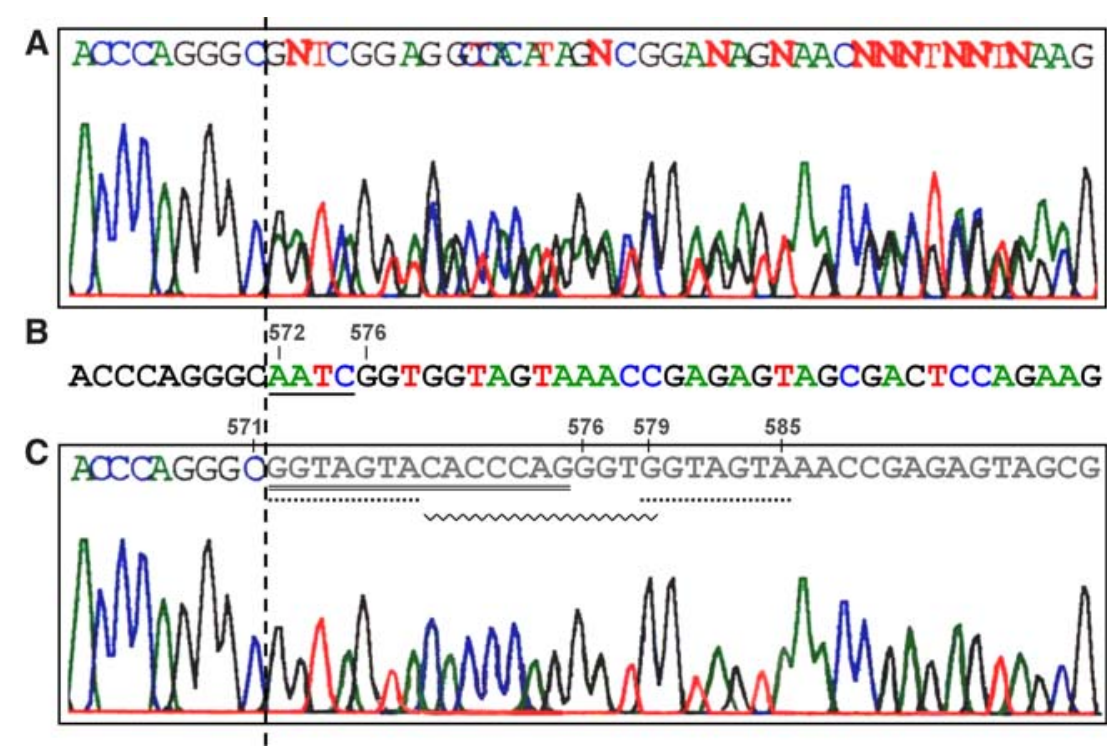

Fig. 1 PAX6 gene mutation analysis of case AN042. A A trace pattern of direct sequencing of the patient DNA. A genomic region including exon 6 was amplified with PCR and subjected to direct sequencing. The sequence in the top line is an output of the automatic sequencer. Since the region with mutation showed mixed pattern of mutated and normal allele $(A)$, curves corresponding to normal sequence $(B)$ was manually removed using an image-editing software Photoshop (Adobe) to visualize only the mutated sequence. $B$ Normal

(Supplemental Fig. 1). This type of mutation was previously reported and included in the Human PAX6 Mutation Database. The aniridia symptom of these patients was mild.

Patient AN021 revealed a novel mutation 786_787ins10, ten-base (CGCAGACGGC) insertion in the sequence between Nt785 and Nt786 (Supplemental Fig. 2B). The inserted ten-base sequence is an exact duplicate of the sequence between Nt776 and Nt785. This mutation results in frameshift and premature termination of translation in the link domain of PAX6 protein.

Patient AN031 of family 3 with apparently sporadic aniridia showed another novel mutation 678_688del11 (Supplemental Fig. 2C), which also causes frameshift and premature termination in the paired domain of PAX6 protein. While no other aniridia patients were found in this family (Supplemental Fig. 1), the mother and greatgrandfather of AN031 had color blindness. However, the relation between PAX6 mutation and color blindness is unclear.

Patient AN041 and her younger brother AN042 revealed another novel mutation, 572_575delAATCins14 (Fig. 1), which results in frameshift and premature termination in the paired domain of PAX6 protein. The patient AN042 presented only aniridia, whereas the patient AN041 was accompanied by VATER association and their mother appeared normal. As a pathogenic mutation for VATER association, A-G point mutation at nucleotide position sequence of the tested region. The four bases with single underline indicate the deleted part of the mutated allele $(C)$. $C$ A trace pattern of the mutant allele generated by eliminating normal sequence. Gray letters show nucleotide sequence determined by manual reading. The 14 bases with double underline indicate the inserted sequence in the mutant allele. Dotted lines show a common sequence between normal and mutant alleles. A wavy line indicates a sequence with a quasipalindromic structure

3,243 of mitochondrial DNA was previously described (Quan and Smith 1972; Damian et al. 1996). However, such point mutation was not detected in the patient AN041 (data not shown). Strabismus was seen in several members of family 4, and it may be related to the same PAX6 mutation.

Database construction

To support understanding of the relationship between PAX6 gene mutation and clinical phenotype, we made a database using the MutationView system (http://mutview.dmb.med.keio.ac.jp/) (Minoshima et al. 2001). A typical screen shot of the MutationView displays cDNA structure (exon 5-exon 13) and protein domains (Supplemental Fig. 3). The height of the bar represents the family number of each mutation that appeared in the literature and the Human PAX6 Mutation Database at MRC. Since novel mutations found in exons 5, 6 and 7 are hard to see in the screen shot, they were highlighted in B.

\section{Discussion}

We have described the PAX6 mutations including three novel types in Japanese aniridia patients. All the analyzed cases revealed deletion, insertion or indel mutation with 
frameshift, resulting in premature termination of translation. Previously, several reports were made regarding Japanese PAX6 mutations mainly for non-typical aniridia or other disease cases, in which missense mutations were predominantly associated (Azuma et al. 1996, 1999, 2003; Sonoda et al. 2000; Yasuda et al. 2002). Two of these, V7D (exon 5a) and S363P, were associated with Peters anomaly, but not aniridia (Azuma et al. 1999, 2003). R125C mutation, first reported from Japan, caused isolated foveal hypoplasia (Azuma et al. 1996). Sonoda et al. reported P118R mutation, which is associated with congenital nystagmus, but not aniridia (Sonoda et al. 2000). Another report by Yasuda et al. described three types of mutations (590ins7, 790del4 and R203X) in five Japanese aniridia patients, all of which are frameshift or nonsense mutations, resulting in premature termination in the paired or link domain of PAX6 protein (Yasuda et al. 2002). Our present results confirmed a general tendency that frameshift/nonsense mutations of PAX6 are frequently associated with aniridia, whereas missense mutations are often found in non-aniridia cases (van Heyningen et al. 2002).

Of three novel mutations, the mutation 572_575delAATCins 14 found in the patients AN041 and AN042 is unique and interesting. The 7-base sequence (GGTAGTA) of the 11-base insertion is exactly same as the sequence 579-585, while the 11-base insertion surrounded by those 7-base sequences shows a quasi-palindromic structure (Fig. 1c). These unique sequence characteristics may have some relation to generating unusual type of mutation.

We have reported an aniridia case AN041 accompanied by VATER association (Quan and Smith 1972; Damian et al. 1996). To our knowledge, this is the first report of an aniridia case accompanied by VATER association.

The area affected with VATER association is generally derived from the mesoderm or endoderm, while aniridia is a disorder of the iris that is in the lineage of the ectoderm. Since the PAX6 gene is generally known to function in differentiating neuronal cells derived from the ectoderm, it is understandable that aniridia is caused by PAX6 mutation. However, we do not know whether VATER association is caused by the detected PAX6 mutation or not.

The database MutationView has various functions including graphical display of all mutations in the genomic and cDNA sequences and protein amino acid sequence with functional domain, frequency of each mutation, or their change by mutation, and functions to classify mutations such as disease symptom and inheritance pattern (Supplemental Fig. 3). This database should be useful to overview PAX6 mutations and genotype-phenotype correlation (Minoshima et al. 2001).

In conclusion, this study added three novel types of mutations that are associated with the development of aniridia.

Acknowledgments This work was supported in part by a Grant-inAid for Scientific Research on Priority Areas from the Ministry of Education, Culture, Sports, Science and Technology (MEXT) and a Grant-in-Aid for Publication of Scientific Research Results from the Japan Society for the Promotion of Science (JSPS).

\section{References}

Azuma N, Nishina S, Yanagisawa H, Okuyama T, Yamada M (1996) PAX6 missense mutation in isolated foveal hypoplasia. Nat Genet 13:141-142

Azuma N, Yamaguchi Y, Handa H, Hayakawa M, Kanai A, Yamada M (1999) Missense mutation in the alternative splice region of the PAX6 gene in eye anomalies. Am J Hum Genet 65:656-663

Azuma N, Yamaguchi Y, Handa H, Tadokoro K, Asaka A, Kawase E, Yamada M (2003) Mutations of the PAX6 gene detected in patients with a variety of optic-nerve malformations. Am J Hum Genet 72:1565-1570

Damian MS, Seibel P, Schachenmayr W, Reichmann H, Dorndorf W (1996) VACTERL with the mitochondrial np 3243 point mutation. Am J Med Genet 62:398-403

Elsas FJ, Maumenee IH, Kenyon KR, Yoder F (1997) Familial aniridia with preserved ocular function. Am J Ophthal 83:718724

Glaster T, Walton DS, Maas RL (1992) Genomic structure, evolutionary conservation and aniridia mutations in the human PAX6 gene. Nature Genet 2:232-239

van Heyningen V, Williamson KA (2002) PAX6 in sensory development. Hum Mol Genet 11:1161-1167

Minoshima S, Mitsuyama S, Ohtsubo M, Kawamura T, Ito S, Shibamoto S, Ito F, Shimizu N (2001) The KMDB/MutationView: a mutation database for human disease genes. Nucl Acids Res 29:327-328

Prosser J, van Heyningen V (1998) PAX6 mutations reviewed. Hum Mutat 11:93-108

Quan L, Smith DW (1972) The VATER association: vertebral defects, anal atresia, tracheoesophageal fistula with esophageal atresia, radial dysplasia. Birth Defects Orig Art Ser VIII(2):7578

Sonoda S, Isashiki Y, Tabata Y, Kimura K, Kakiuchi T, Ohba N (2000) A novel PAX6 gene mutation (P118R) in a family with congenital nystagmus associated with a variant form of aniridia. Graefes Arch Clin Exp Ophthalmol 238:552-558

Ton CC, Hirvonen H, Miwa H, Weil MM, Monaghan P, Jordan T, van Heyningen V, Hastie ND, Meijers-Heijboer H, Drechsler M (1991) Positional cloning and characterization of a paired boxand homeobox-containing gene from the aniridia region. Cell 67:1059-1074

Yasuda T, Kajimoto Y, Hori M (2002) PAX6 mutation as a genetic factor common to aniridia and glucose intolerance. Diabetes $51: 224-230$ 